\title{
Smartphone e folder podem ser uma alternativa para reduzir o comportamento sedentário? Estudo piloto
}

\author{
Can smartphone and folder be an alternative to reduce sedentary behavior? \\ Pilot study
}

\section{AUTORES \\ Douglas de Assis Teles Santos ${ }^{1}$ (D) \\ Lucas Lima Galvão² (D) \\ Rafaela Gomes dos Santos $^{1}$ (D) \\ Ricardo Borges Viana ${ }^{3}$ (D) \\ Emille Camila de Oliveira Santos ${ }^{1}$ (D) \\ Rízia Rocha Silva² (i) \\ Sheilla Tribess $^{2}$ (D) \\ Jair Sindra Virtuoso Junior² (DD \\ Claudio Andre Barbosa de Lira $^{3}$ (D) \\ 1 Universidade do Estado da Bahia, Colegiado de Educação Física, Teixeira de Freitas, Bahia, Brasil. \\ 2 Universidade Federal do Triângulo Mineiro, Departamento de Ciências do Esporte, Uberaba, Minas Gerais, Brasil. \\ 3 Universidade Federal de Goiás, Faculdade de Educação Física e Dança, Goiânia, Goiás, Brasil.}

\section{CONTATO}

Douglas de Assis Teles Santos

datsantos@uneb.br

Avenida Kaikan s/n, Setor universitário,

Teixeira de Freitas, Bahia, Brasil.

CEP: 45992-255.

DOI

10.12820/rbafs.26e0191

\section{(cc) BY}

Este trabalho está licenciado com uma Licença Creative Commons - Atribuição 4.0 Internacional.

\begin{abstract}
RESUMO
O objetivo do estudo foi investigar se a exposição a informações sobre vida saudável, via aplicativo de mensagem de smartphone ou folder impresso, reduz o comportamento sedentário (CS) de universitários. Este estudo é um piloto prospectivo quase experimental, constituído por 69 participantes, randomizados por turma, em dois grupos de intervenção via mensagem de texto Whatsapp (grupo MSG) e folder impresso (grupo FOLDER). As intervenções apresentaram orientações semelhantes com foco geral de promover um perfil de vida saudável, reduzindo o CS. As mensagens foram encaminhadas às segundas-feiras por um período de quatro semanas consecutivas. O tempo exposto ao CS foi avaliado a partir do Questionário Internacional de Atividade Física na Baseline e após quatro semanas de intervenção. Para avaliar o efeito do tempo, da intervenção e a interação (tempo*intervenção) no CS, foram utilizados modelos mistos generalizados para medidas repetidas, utilizando uma matriz de covariância autorregressiva de primeira ordem (AR1) e foi utilizado o teste post hoc de Bonferroni. Foi adotado o nível de significância de 5\%. Foram observados efeitos significantes das intervenções ( $F=5,30 ; \mathrm{p}$ $=0,024)$, FOLDER $(519,71 \pm 28,01)$ e MSG $(430,37 \pm 26,82)$, e dos tempos $(\mathrm{F}=19,05 ; \mathrm{p}<0,001)$ baseline $(522,07 \pm 22,18)$ e após 4 semanas $(428,00 \pm 22,18)$ mas não foi observado interação entre o tempo e a intervenção $(F=0,430 ; p=0,514)$. Foram observadas diferenças significantes entre os grupos no momento pós intervenção $(\mathrm{p}=0,022)$. Ambas as intervenções parecem ser úteis para a redução do tempo exposto ao CS em uma população de adultos jovens durante o período de quatro semanas.
\end{abstract}

Palavras-chave: Comportamento Sedentário; Tecnologia; Saúde.

\section{ABSTRACT}

The objective of the study was investigate whether exposure to information about healthy living, via smartphone message application or printed folder, reduces the sedentary behavior (SB) of university students. This study is an almost experimental prospective pilot, consisting of 69 participants, randomized by class, in two intervention groups via Whatsapp text message (MSG group) and printed folder (FOLDER group). The interventions presented similar guidelines with a general focus on promoting a healthy life profile, reducing $S B$. The messages were forwarded on Mondays for a period of four consecutive weeks. The time exposed to the $S B$ was evaluated using the International Physical Activity Questionnaire on the baseline and after four weeks of intervention. To evaluate the effect of time, intervention and interaction (time *intervention) in the $S B$, generalized mixed models for repeated measures were used, using a first order autoregressive covariance matrix (AR1) and Bonferroni post hoc test was used. The significance level of 5\% was adopted. Significant effects of the interventions $(F=5.30 ; p=0.024), F O L D E R(519.71 \pm 28.01)$ and $M S G(430.37 \pm 26.82)$, and of the times $(F=19.05 ; p<0.001)$ baseline $(522.07 \pm 22.18)$ and after 4 weeks $(428.00 \pm 22.18)$ but no interaction was observed between time and intervention $(F=0.430 ; p=0.514)$. Significant differences were observed between the groups at the time after the intervention $(p=0.022)$. Both interventions appear to useful for reducing the time exposed to $S C$ in a population of young adults over the four-week period.

Keywords: Sedentary behavior; Technology; Health.

\section{Introdução}

O comportamento sedentário (CS) conceituado como o comportamento realizado em estado de vigília, na posição sentada, deitada ou reclinada com gasto energético $\leq 1,5 \mathrm{METs}^{1}$, vem ganhando atenção constante da comunidade científica nos últimos anos, com diversos estudos demonstrando seus malefícios à saúde de maneira geral, como fatores de risco cardiometabólicos, diabetes tipo II, doenças cardiovasculares, obesidade, depressão, alguns tipos de canceres e morte prematura por todas as causas ${ }^{2,3}$. A ocorrência desses efeitos deletérios à saúde parece ser independente do nível de atividade física ${ }^{3}$. Estudos recentes vêm demonstrando que interrupções regulares no CS estão associadas a resul- 
tados benéficos à saúde cardiometabólica ${ }^{4}$, evidenciando que interrupções do tempo sentado com breves períodos de caminhada de intensidade leve ou moderada promovem benefícios sobre as respostas pós-prandiais de glicose e insulina ${ }^{5}$.

Esses achados ressaltam a necessidade de intervenções nas quais os participantes são alertados sobre os efeitos de permanecer em CS por prolongado período de tempo e tais intervenções devem encorajar as pessoas a interromper este comportamento ${ }^{6}$. Além disso, as intervenções devem ser acessíveis e fáceis de implementar na maioria dos ambientes ${ }^{7}$.

Os smartphones tornaram-se ferramentas populares para adotar um estilo de vida saudável porque constituem alternativas potencialmente de baixo custo para alcançar muitos indivíduos em um período mais longo ${ }^{8}$ e por apresentar um importante papel no cotidiano das pessoas, sendo atrativo à diferentes gerações 9 .

Em pesquisa realizada nos Estados Unidos, foi demonstrado que $96 \%$ dos estudantes universitários possuem telefone celular ${ }^{10}$. Fennell et al. ${ }^{11}$, em estudo de revisão, demonstram que estudantes universitários usam smartphones em média 279-528 minutos por dia, sendo está uma fase de grandes mudanças na vida dos jovens adultos, onde comportamentos de risco devem ser evitados. Em recente relatório publicado entre as empresas We Are Social e Hoostsuit ${ }^{12}$, identificaram que no Brasil a taxa de utilização de aparelho de celular chega à 97\%, cerca de 205 milhões de pessoas e destas $71 \%$ (150 milhões) tem acesso à internet, e 66\% (140 milhões) utiliza mídias sociais, onde as mais utilizadas são Youtube, Facebook e Whatsapp representando respectivamente 96\%, 90\% e 88\% de utilização entre os 16 e 64 anos. Ainda segundo o mesmo relatório, o brasileiro permanece em média 9 horas utilizando a internet e 3 horas utilizando as mídias sociais, e as pessoas entre 18 e 34 anos são as que mais utilizam as mídias sociais.

O termo "mídia social" geralmente se refere a ferramentas baseadas na Internet que permitem que indivíduos e comunidades compartilhem ou troquem informações, notícias, ideias, vídeos, imagens, mensagens pessoais e outros conteúdos em tempo real ou não real ${ }^{13}$. $\mathrm{O}$ alto uso de mídias sociais está associado a um maior tempo em $\mathrm{CS}^{11}$, menor número de passos e maior consumo de calorias por dia ${ }^{14}$. Além disto, pessoas em elevado tempo de utilização do smartphone apresentam maior quantidade de massa gorda e menor quantidade de massa muscular ${ }^{14}$.

Em recente estudo de meta-análise, com objetivo de determinar a associação entre o uso de smartphone e estresse e ansiedade, incluiu 37 estudos com participantes da Europa, Ásia e América do Norte, onde os autores observaram uma associação entre o uso de telefone celular e estresse e ansiedade. Adicionalmente, os autores constataram o ano de publicação do estudo como moderador significante, indicando que a relação entre o uso do smartphone e o estresse e ansiedade se fortaleceu ao longo do período dos estudos publicados $(2007-2017)^{15}$.

Entretanto, pesquisadores vêm pesquisando intervenções eficientes de reduzir o tempo em CS tentativa de desenvolver maneiras eficientes de reduzir o tempo em CS com a utilização de smartphones ${ }^{6,7,16}$,exergames $^{17}$ e quebras ativas do $\mathrm{CS}^{18}$, em diversas populações. Uma meta-análise realizada por Direito et al. ${ }^{19}$, incluiu 21 ensaios clínicos randomizados, sendo que 7 dessas incluíram aplicativos de saúde. Os autores encontraram que as intervenções em saúde móvel levaram à diminuição do tempo exposto ao CS em comparação ao grupo controle, indicando que o smartphone pode ser estratégia viável de redução do tempo em CS.

Com aumento na utilização de smartphones, intervenções baseadas em tecnologias de informação estão amplamente disponíveis em formatos de aplicativos fitness e de nutrição apresentando grande adesão, porém com elevada desistência ${ }^{20}$, demonstrando sucessos variados e seus efeitos por períodos limitados ${ }^{21}$. Desta forma, estudos têm sido desenvolvidos buscando melhor aderência e efetividade para redução do CS, incluindo aqueles com utilização de exergames em diversos subgrupos populacionais ${ }^{17,22}$.

Com os enunciados aqui apresentados, partimos da hipótese que o aplicativo já aceito e bastante utilizado de mensagem Whatsapp, pudesse ser ferramenta útil de redução ao CS, desta forma preenchendo a lacuna na literatura sobre a possível efetividade deste aplicativo no CS. O estudo teve o objetivo de investigar se a exposição a informações sobre vida saudável, via aplicativo de mensagem de smartphone ou folder impresso, reduz o comportamento sedentário (CS) de universitários.

\section{Métodos}

Este estudo é um piloto prospectivo quase experimental, constituído por 69 participantes, randomizados por turma, em dois grupos de intervenção via mensagem de texto Whatsapp (grupo MSG) e folder impresso (grupo FOLDER). Os participantes foram recrutados entre os estudantes do curso de Educação Física da Universida- 
de do Estado da Bahia (Campus X, Teixeira de Freitas, Brasil), entre os meses de abril e junho de 2017. Os discentes de quatro turmas foram informados do tempo, duração e dos objetivos do estudo e foram convidados a participarem do projeto, sendo este recrutamento realizado de forma verbal pelos pesquisadores em contato coletivo por turma. Aqueles que concordaram em participar assinaram o termo de consentimento livre e esclarecido. Todos os procedimentos estavam de acordo com padrões éticos do comitê institucional (2.961.296) e com a declaração de Helsinque.

Os participantes eram elegíveis se: estivessem devidamente matriculados no curso presencial de Educação Física, presença regular na instituição, possuíssem dispositivo smartphone com a utilização do Whatsapp e deveriam participar das avaliações da baseline e pós intervenção de quatro semanas. Foram excluídos participantes com informações incompletas e qualquer ausência, no grupo FOLDER, nos dias de sua entrega. Os participantes foram avaliados no baseline e no pós intervenção de quatro semanas, sendo os mesmos alocados, randomicamente por sorteio, por período letivo do curso para evitar que um participante de determinada intervenção pudesse ter influência sobre outro (Figura 1).

Após o primeiro contato com os participantes foram obtidas informações de idade, estatura, massa corporal, CS e telefone para contato que estivesse registrado com o Whatsapp. O foco geral das intervenções foi reduzir o CS. A intervenção FOLDER (suplemento 1) foi composta por folheto ilustrado e colorido descrevendo 10 passos visando a redução do CS (1. Faça pausas regulares, ficando em pé a cada 30 minutos; 2 . Subir escadas ao invés do elevador; 3. Fique de pé enquanto estiver ao telefone; 4. Caminhe para falar com seu colega ao invés de utilizar MSG; 5. Coloque a lixeira longe da mesa; 6. Limite o tempo de tela; 7. Beba bastante água; 8. Caminhe com um amigo; 9. Leve seu bicho de estimação para passear; 10 . Alongue-se.), incentivando atividades da vida diária e atividade leves para substituir o CS, esse folheto foi entregue as segundas-feiras por um período de quatro semanas, sempre no período de aulas da turma (vespertino ou matutino).

$\mathrm{Na}$ intervenção MSG (suplemento 2) a mensagem foi redigida com as mesmas informações com a utilização de emojis, de modo que aumentasse a atração e entendimento da intervenção, e enviadas como lista de transmissão do aplicativo Whatsapp. As mensagens eram encaminhadas às segundas-feiras por um período de quatro semanas no período de aulas das turmas

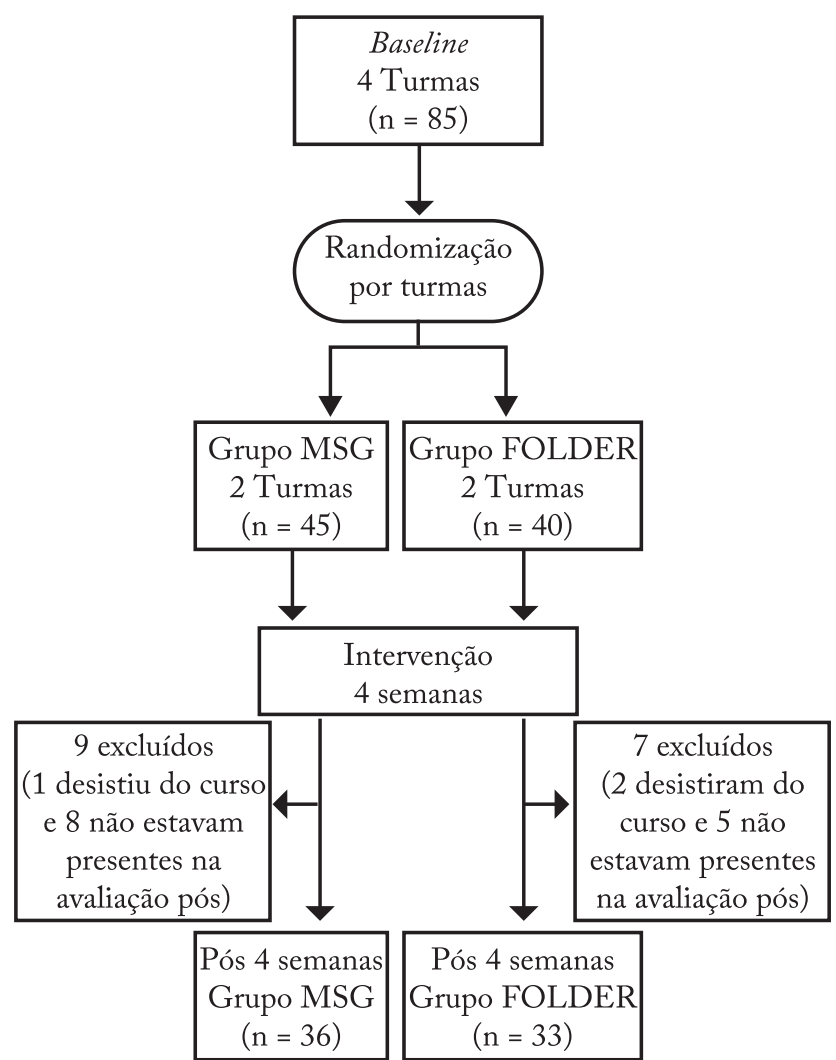

Figura 1 - Delineamento do estudo. Teixeira de Freitas, Bahia, 2020.

(vespertino ou matutino). Ambas as intervenções foram baseadas em estratégia elaborado pelo grupo on your feet britain (10 way to sit less at work $)^{23}$.

Após o período de intervenção de quatro semanas novamente foi mensurado o tempo de CS de ambos os grupos no dia pré-determinado, de modo que em ambos os grupos as medidas fossem mensuradas no mesmo dia.

Foram avaliadas medidas antropométricas de estatura e massa corporal (MC) avaliados por meio de uma balança da marca Filizola (Micheletti MIC-200PP, Brasil), sendo calculado o índice de massa corporal (IMC) a partir da equação $\mathrm{IMC}=\mathrm{MC} /\left(\mathrm{EST}^{2}\right)$.

O CS foi determinado pelo tempo exposto na posição sentada, avaliado a partir das questões de tempo sentado em um dia habitual de semana ("Quanto tempo no total, você gasta sentado(a) durante um DIA DE SEMANA?") e um dia habitual de fim de semana ("Quanto tempo no total, você gasta sentado(a) durante um DIA DE FINAL SEMANA?"), de acordo a sessão cinco do Questionário Internacional de Atividade Física (IPAQ) versão longa, adaptado para população brasileira ${ }^{24}$. O tempo exposto ao CS, minutos/ dia, foi determinado a partir da média ponderada do 
tempo sentado em um dia de semana e um dia de final de semana: [(tempo sentado em um dia de semana $\mathrm{x} 5$ + tempo sentado em um dia de final de semana $\mathrm{x}$ 2) / 7]. A avaliação do CS foi realizada pré e pós (4 semanas) intervenção.

Para o banco de dados foi utilizado o software Epidata 3.1b (EpiData Association, Dinamarca) e as análises conduzidas no SPSS versão 23.0 (IBM, EUA). Foram utilizados procedimentos da estatística descritiva, média e desvio padrão (DP) para estatura e mediana e intervalo interquartil (IIQ) das variáveis MC e IMC, de modo caracterizar a amostra. Para os procedimentos inferenciais foram utilizados, o teste de normalidade Shapiro Wilk e em seguida utilizados teste $t$ de amostras independentes para estatura e teste $U$ de $M a n n-W b t i-$ ney para MC e IMC. O $\Delta$ absoluto foi calculado subtraindo o tempo exposto ao CS do momento Pré pelo momento Pós.

Para avaliar o efeito do tempo, da intervenção e a interação (tempo*intervenção) no CS, foram utilizados modelos mistos generalizados para medidas repetidas, utilizando uma matriz de covariância autorregressiva de primeira ordem (AR1), pois, com as intervenções é esperado uma redução do tempo exposto ao CS, sendo utilizado o teste post hoc de Bonferroni. Foi adotado o nível de significância de 5\%.

\section{Resultados}

Ao final do período de 4 semanas de intervenção, um total de 16 pessoas foram excluídas ( 5 desistiram do curso e 11 não compareceram à avaliação). A amostra final foi composta por 69 participantes de ambos os sexos com amplitude etária de 18 a 54 anos, sendo destes, 45 mulheres $(22,7 \pm 5,4$ anos) e 24 homens ( $28,0 \pm$ 9,9 anos), randomizados em dois grupos sendo 36 no grupo Mensagem de Whatsapp (MSG) e 33 no grupo FOLDER. A Tabela 1 apresenta as características antropométricas dos participantes quanto a intervenção.

A Figura 2 apresenta a distribuição do tempo exposto ao CS de ambos os grupos ao longo da intervenção. Quanto a comparação do modelo misto generalizado, foram observados efeitos significantes das intervenções ( $\mathrm{F}=5,30 ; \mathrm{p}=0,024)$ FOLDER $(519,71$ $\pm 28,01)$ e $\mathrm{MSG}(430,37 \pm 26,82)$, e dos tempos $(\mathrm{F}=$ $19,05 ; \mathrm{p}<0,001)$ baseline $(522,07 \pm 22,18)$ e após 4 semanas de intervenção $(428,00 \pm 22,18)$, mas não foram observadas interações entre o tempo e a intervenção ( $F$ $=0,430 ; p=0,514)$. Foram observadas diferenças significantes entre os grupos no momento pós intervenção $(\mathrm{p}=0,022)$, mas não no momento pré $(\mathrm{p}=0,093)$.

Os deltas absolutos e relativos médios referentes aos tempos de exposição ao CS estão apresentados na Tabela 2.

Tabela 1 - Comparação das características antropométricas dos participantes nos grupos MSG e FOLDER. Teixeira de Freitas, Bahia, $2020(n=69)$.

\begin{tabular}{lcccc}
\hline & Total & MSG & FOLDER & p \\
\hline Estatura $(\mathrm{m})$ & $1,66(0,11)$ & $1,68(0,09)$ & $1,67(0,08)$ & 0,863 \\
MC $(\mathrm{kg})$ & $60,0(1,59)$ & $63,0(2,31)$ & $60,0(1,91)$ & 0,482 \\
$\operatorname{IMC}\left(\mathrm{kg} / \mathrm{m}^{2}\right)$ & $22,6(0,45)$ & $23,1(0,67)$ & $21,8(0,53)$ & 0,394 \\
\hline
\end{tabular}

$\mathrm{MC}=$ massa corporal; $\mathrm{IMC}$ = índice de massa corporal. Dados expresso em mediana e intervalo interquartil, exceto para estatura que são expressos em média e desvio padrão.

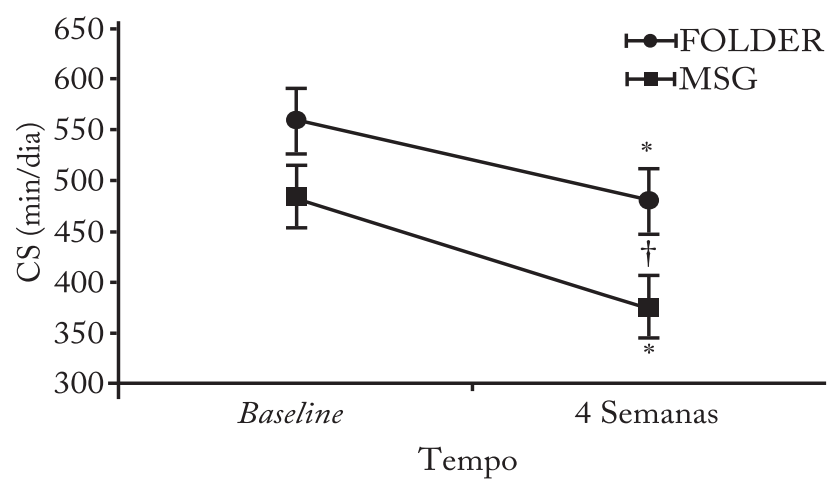

Figura 2 - Distribuição e diferenças das intervenções da baseline e após quatro semanas de intervenção. Teixeira de Freitas, Bahia, 2020 $(\mathrm{n}=69)$.

* Diferença significante em relação aos valores da baseline ( $\mathrm{p}<$ $0,05)$. †Diferença significante entre os grupos no momento pós intervenção de quatro semanas $(\mathrm{p}=0,022)$.

Tabela 2 - Deltas absolutos e relativos médios do comportamento sedentário após as quatro semanas das intervenções de MSG e FOLDER. Teixeira de Freitas, Bahia, $2020(n=69)$.

\begin{tabular}{lcccc}
\hline & $\begin{array}{c}\text { Pré } \\
(\mathrm{min} / \mathrm{dia})\end{array}$ & $\begin{array}{c}\text { Pós } \\
(\mathrm{min} / \mathrm{dia})\end{array}$ & $\begin{array}{c}\Delta \text { absoluto } \\
(\mathrm{min} / \mathrm{dia})\end{array}$ & $\begin{array}{c}\Delta \text { relativo } \\
(\%)\end{array}$ \\
\hline MSG & 484,47 & 376,27 & 108,20 & 22,33 \\
FOLDER & 559,68 & 479,75 & 79,93 & 14,28 \\
\hline
\end{tabular}

$\Delta=$ delta $;$ MSG = grupo mensagem de Whatsapp FOLDER $=$ grupo folder. $\mathrm{O} \Delta$ absoluto foi calculado subtraindo o tempo exposto ao comportamento sedentário do momento Pré pelo momento Pós. Dados expressos em média e desvio padrão.

\section{Discussão}

$\mathrm{O}$ presente estudo teve como objetivo investigar se a exposição a informações sobre vida saudável, via aplicativo de mensagem de smartphone ou folder impresso, reduz o CS de universitários. Foi possível após quatro semanas de intervenção observar que em ambos protocolos obtiveram diminuição do tempo exposto ao CS. 
Visto o grande número de agravos que o CS pode ocasionar para a saúde de maneira geral ${ }^{3}$, torna-se importante avaliar maneiras eficientes de diminuir o tempo exposto a este comportamento, por isso diversos estudos têm investigado maneiras de alcançar este objeti$\mathrm{vo}^{6,7,14,16,22,25}$. Os nossos resultados sugerem que o smartphone é uma alternativa eficaz para mudança do estilo de vida. Considerando que o smartphone tem custo relativamente baixo e que o seu uso é amplamente disseminado na população, o uso dos smartphones para estratégias como a adotada pelo presente estudo são promissoras.

Em nosso estudo foi observado diminuição média de $94 \mathrm{~min} /$ dia de CS em ambas intervenções, esses resultados são superiores aos encontrados em outros es$\operatorname{tudos}^{6,7}$. Arrogi et al. ${ }^{6}$ utilizaram uma intervenção baseada em aplicativo de smartphone (stAPP) em adultos belgas com objetivo de diminuir o tempo exposto ao CS com pausas, e obtiveram resultados de diminuição de $30 \mathrm{~min} /$ dia de CS durante a semana. Bond et al. ${ }^{7}$ testaram três intervenções de quebra do CS (i. $3 \mathrm{~min}$ de quebra a cada 30 minutos sentado continuamente; ii. 6 min de quebra a cada 60 minutos de tempo sedentário; iii. 12 min de pausas a cada 120 minutos em CS), e observaram diminuição significante em ambas intervenções em relação a baseline durante um período de quatro semanas, entretanto, destacaram que interrupções mais curtas, por menor tempo em CS são duas vezes mais eficientes que quebras mais longas em CS prolongado. A intervenção destacada neste estudo consiste em promover pequenas mudanças comportamentais ao longo do dia.

Para a intervenção com base em mensagem de texto via aplicativo de smartphone, houve uma tendência de ser mais eficaz em relação ao FOLDER impresso, com a redução média de $22,3 \%$ vs 14,3\% do tempo exposto ao $\mathrm{CS}$, respectivamente. Esse fato pode ser atribuído ao smartphone estar cada vez mais integrado à sociedade, ser de fácil acessibilidade e estar cada vez mais presente no cotidiano das pessoas e com o aumento do seu uso nos últimos anos, tem gerado muita atenção para incorporá-lo como ferramenta para melhorar a saúde ${ }^{26}$. As tecnologias de smartphone têm se mostrado viáveis e aceitáveis para modificar o CS de adultos e idosos ${ }^{16} \mathrm{e}$ mulheres sedentárias ${ }^{25}$.

Com os avanços tecnológicos, o aumento do uso dos smartphones na população em geral e menores custos com sua utilização têm-se mostrado mais atrativo como ferramenta de combate ao tempo sedentário. A redução do tempo exposto com o FOLDER impresso também se mostrou significante ao longo de quatro semanas.

Estudo piloto randomizado de 8 semanas com 29 mulheres afro-americanas, alocadas em dois grupos, um que recebeu uma intervenção baseada na Teoria Social Cognitiva entregue via Facebook e mensagem de texto e outro grupo baseado em impressão padrão, com objetivo de promover a atividade física e consequentemente reduzir o tempo exposto ao CS, observaram resultados conflitantes ao nosso estudo, com reduções em $71 \mathrm{~min} / \mathrm{sem}$ no grupo que recebeu a intervenção via Facebook e um aumento de $118 \mathrm{~min} / \mathrm{sem}$ no grupo que recebeu a intervenção via impressão padrão ${ }^{27}$.

Outro estudo que objetivou aumentar o nível de atividade física de mulheres insuficientemente ativas, observaram reduções de $33,3 \mathrm{~min} / \mathrm{sem}$ no tempo sentado no grupo de intervenção vs $1 \mathrm{~min} / \mathrm{sem}$ de redução no grupo placebo. A intervenção consistia em folha de informação sobre recursos disponíveis na própria comunidade investigada e um livreto impresso que correspondia a metas que cada indivíduo deveria alcançar ${ }^{28}$.

Como limitações é possível destacar a falta de um grupo controle de modo a comparar os momentos das intervenções e ajustar possíveis erros sistemáticos. Não foi possível realizar o acompanhamento pós-intervenção ao longo do tempo para verificar como comporta-se o tempo de exposição ao CS, se os valores continuam a declinar ou após intervenção eles se elevam. Não foi avaliada ainda a viabilidade da intervenção quanto à satisfação. Entretanto, Lewis et al. ${ }^{29}$ destacam a importância desta avaliação através do modelo RE-AIM ${ }^{30}$, sendo possível estabelecer métricas de efetividade, adesão e satisfação dos participantes quanto a intervenção, e por se tratar de uma população de universitários de curso da área da saúde, pode ter contribuído para diminuição deste tempo de exposição ao CS por se tratar de assunto abordado dentro da graduação. Outra limitação é o fato de algumas pessoas não possuírem acesso remoto a internet, entretanto isso foi atenuado pelo fator de que as intervenções eram encaminhadas no período de letivo, cujo acesso à internet era aberto. Como ponto forte, podemos destacar a utilização de intervenções de baixo custo, com utilização de smartphone e um aplicativo já bastante utilizado e aceito de MSG pela população estudada, tornando-o de fácil acesso e demonstrando boa efetividade e baixo custo. E uma população universitária, que constitui um grupo em médio consistentemente exposto a longos períodos sedentário.

Ambas as intervenções parecem ser úteis para a redução do tempo exposto ao CS em uma população de 
adultos jovens durante o período de quatro semanas. Apesar de não haver ponto de corte para o CS em recomendações para a saúde, acreditamos que as reduções encontradas em ambos os grupos são benéficas e postulamos como desafio a substituição do tempo exposto ao CS por atividades físicas. Este estudo fornece informações importantes para orientar o desenvolvimento de futuras intervenções focadas no sedentarismo, mostrando que encorajar os indivíduos a interromper o CS mais frequentemente com orientações simples pode ser eficaz. Mais pesquisas são necessárias para determinar a eficácia a longo prazo, com maior amostra populacional, inclusão de grupo controle, acompanhamento por maior período, incluindo período pós intervenção, de modo a estimar a eficiência da mesma por longos períodos.

\section{Conflito de interesse}

Os autores declaram não haver conflito de interesses.

\section{Contribuição dos autores}

Santos DAT e Galvão LL contribuíram significativamente na concepção e desenho do estudo, na análise e interpretação dos dados, e substancialmente na elaboração do artigo, na revisão crítica do conteúdo intelectual do artigo e na aprovação da versão final. Santos RG, Viana RB, Tribess S, Virtuoso Junior JS e Lira $\mathrm{CAB}$ contribuíram na elaboração do artigo, da revisão crítica do conteúdo e na aprovação da versão final. Santos ECO e Silva RR contribuíram no e desenho do estudo, na elaboração inicial, análise e interpretação dos dados e na aprovação da versão final.

\section{Agradecimentos}

Os autores agradecem à Universidade do Estado da Bahia e a todos os participantes do estudo.

\section{Referências}

1. Tremblay MS, Aubert S, Barnes JD, Saunders TJ, Carson V, Latimer-Cheung AE, et al. Sedentary Behavior Research Network (SBRN) - Terminology Consensus Project process and outcome. Int. J. Behav. Nutr. Phys. Act. 2017;14(1):1-17.

2. Santos DAT, Virtuoso JS, Meneguci J, Sasaki JE, Tribess S. Combined Associations of Physical Activity and Sedentary Behavior With Depressive Symptoms in Older Adults. Issues Ment. Health Nurs. 2017;38(3):272-6.

3. Owen N, Healy GN, Matthews CE, Dunstan DW. Too much sitting: The population health science of sedentary behavior. Exerc. Sport Sci. Rev. 2010;38(3):105-13.

4. Benatti FB, Ried-Larsen M. The Effects of Breaking up Prolonged Sitting Time: A Review of Experimental Studies. Med. Sci. Sports Exerc. 2015;47(10):2053-61.

5. Dunstan DW, Kingwell BA, Larsen R, Healy GN, Cerin E, Hamilton MT, et al. Breaking up prolonged sitting reduces postprandial glucose and insulin responses. Diabetes Care. 2012;35(5):976-83.
6. Arrogi A, Bogaerts A, Seghers J, Devloo K, Vanden Abeele V, Geurts L, et al. Evaluation of stAPP: A smartphone-based intervention to reduce prolonged sitting among Belgian adults. Health Promot. Int. 2019;34(1):16-27.

7. Bond DS, Thomas JG, Raynor HA, Moon J, Sieling J, Trautvetter J, et al. B-MOBILE - A smartphone-based intervention to reduce sedentary time in overweight/obese individuals: A within-subjects experimental trial. PLoS One. 2014;9(6):1-8.

8. King AC, Hekler EB, Grieco LA, Winter SJ, Sheats JL, Buman MP, et al. Harnessing Different Motivational Frames via Mobile Phones to Promote Daily Physical Activity and Reduce Sedentary Behavior in Aging Adults. PLoS One. 2013;8(4):1-8.

9. Silva SR. "Eu Não Vivo Sem Celular": Sociabilidade, Consumo, Corporalidade e Novas Práticas nas Culturas Urbanas. Intexto, Porto Alegre. 2007;2(17):1-17.

10. Smith A, Rainie L, Zickuhr K. College students and technology. Pew Research; 2011. Disponível em: <https:// www.pewresearch.org/internet/2011/07/19/collegestudents-and-technology/> [2020 novembro].

11. Fennell C, Barkley JE, Lepp A. The relationship between cell phone use, physical activity, and sedentary behavior in adults aged 18-80. Comput. Human Behav. 2019;90(1):53-9.

12. Kemp S.Digital 2020: Brazil. DataReportal; 2020. Disponível em: <https://datareportal.com/reports/digital-2020-brazil> [2020 novembro].

13. Lambert, KM, Barry, P, Stokes, G. Risk management and legal issues with the use of social media in the healthcare setting. J. Healthc Risk Manag. 2012;31(4):41-7.

14. Kim SE, Kim JW, Jee YS. Relationship between smartphone addiction and physical activity in Chinese international students in Korea. J. Behav. Addict. 2015;4(3):200-5.

15. Vahedi Z, Saiphoo A. The association between smartphone use, stress, and anxiety: A meta-analytic review. Stress Heal. 2018;34(3):347-58.

16. Direito A, Tooley M, Hinbarji M, Albatal R, Jiang Y, Whittaker R, et al. Tailored Daily Activity: An Adaptive Physical Activity Smartphone Intervention. Telemed. e-Health. 2019;26(4):1-12.

17. Baranowski T, Baranowski J, Chen T-A, Buday R, Beltran A, Dadabhoy H, et al. Videogames That Encourage Healthy Behavior Did Not Alter Fasting Insulin or Other Diabetes Risks in Children: Randomized Clinical Trial. Games Health J. 2019;8(4):257-64.

18. Ee J, Parry S, Oliveira BIR de, McVeigh JA, Howie E, Straker L. Does a classroom standing desk intervention modify standing and sitting behaviour and musculoskeletal symptoms during school time and physical activity during waking time? Int. J. Environ. Res. Public Health. 2018;15(8):1-13.

19. Direito A, Carraça E, Rawstorn J, Whittaker R, Maddison R. mHealth Technologies to Influence Physical Activity and Sedentary Behaviors: Behavior Change Techniques, Systematic Review and Meta-Analysis of Randomized Controlled Trials. Ann. Behav. Med. 2017;51(2):226-39.

20. Direito A, Walsh D, Hinbarji M, Albatal R, Tooley M, Whittaker $\mathrm{R}$, et al. Using the Intervention Mapping and Behavioral Intervention Technology Frameworks: Development of an mHealth Intervention for Physical Activity and Sedentary Behavior Change. Heal. Educ. Behav. 2018;45(3):331-48.

21. Broekhuizen K, Kroeze W, Poppel MNM Van, Oenema A, Brug J. A systematic review of randomized controlled trials on the effectiveness of computer-tailored physical activity and dietary behavior promotion programs: An update. Ann. Behav. Med. 2012;44(2):259-86. 
22. Garn AC, Baker BL, Beasley EK, Solmon MA. What are the benefits of a commercial exergaming platform for college students? Examining physical activity, enjoyment, and future intentions. J. Phys. Act. Heal. 2012;9(2):311-8.

23. On Your Feet Britain. 10 ways to sit less at work. 2016; Disponível em: <http://onyourfeetday.com/britain/ downloads $>$ [2020 novembro].

24. Matsudo S, Araújo T, Matsudo V, Andrade D, Andrade E, Oliveira LC, et al. Questionário internacional de atividade física (IPAQ): estudo de validade e reprodutibilidade no Brasil. Rev. Bras. Ativ. Fís. Saúde. 2001;6(2):5-18.

25. Fukuoka Y, Vittinghoff E, Jong SS, Haskell W. Innovation to motivation-pilot study of a mobile phone intervention to increase physical activity among sedentary women. Prev. Med. 2010;51(3-4):287-9.

26. Kamboj AK, Krishna SG. Pokémon GO: An innovative smartphone gaming application with health benefits. Prim. Care Diabetes. 2017;11(4):397-9.
27. Joseph RP, Keller C, Adams MA, Ainsworth BE. Print versus a culturally-relevant Facebook and text message delivered intervention to promote physical activity in African American women: A randomized pilot trial. BMC Womens Health. 2015;15(30):1-18.

28. Lane A, Murphy N, Bauman A. An effort to "leverage" the effect of participation in a mass event on physical activity. Health Promot. Int. 2015;30(3):542-51.

29. Lewis BA, Napolitano MA, Buman MP, Williams DM, Nigg CR. Future directions in physical activity intervention research: expanding our focus to sedentary behaviors, technology, and dissemination. J. Behav. Med. 2017;40(1):112-26.

30. Almeida FA, Brito FA, Estabrooks PA. Modelo RE-AIM: Tradução e Adaptação cultural para o Brasil. Rev. Família, Ciclos Vida e Saúde no Context. Soc. 2013;1(1):6-16.

Recebido: $13 / 10 / 2020$

Aprovado: 10/03/2021

\section{Como citar este artigo:}

Santos DAT, Galvão LL, Santos RG, Viana RB, Santos ECO, Silva RR, Tribess S, Virtuoso Junior JS, Lira CAB. Smartphone e folder podem ser uma alternativa para reduzir o comportamento sedentário? Estudo piloto. Rev Bras Ativ Fís Saúde. 2021;26:e191. DOI: 10.12820/ 